\title{
Factores socioeconómicos de vulnerabilidad en la ciudad de Acapulco, Guerrero, México
}

\section{Social vulnerability factors in the city of Acapulco, Guerrero, Mexico}

\author{
Neftalí García Castro
}

\section{Resumen}

Esta investigación revela los factores socioeconómicos de vulnerabilidad existentes en la ciudad de Acapulco, Guerrero (México). La vulnerabilidad social es un estado de incapacidad humana que se manifiesta de manera colectiva y cuyo origen es multifactorial. En trabajo tiene como objetivo central exponer la vulnerabilidad social como característica territorial neurálgica para formular criterios de prevención y mitigación, valorar el impacto potencial y las acciones posteriores a la ocurrencia de eventos que generan consecuencias negativas. Por lo tanto, se presentan fundamentos conceptuales para describir e identificar las diferencias socioterritoriales de esta ciudad mexicana, con base en el valor índice medio, el cual permitió evaluar el acceso heterogéneo a un conjunto de activos y estructura de oportunidades que determinan las condiciones de vulnerabilidad social de la población examinada. De esta manera, se detectó que la mayor parte de los acapulqueños carecen de condiciones socioeconómicas que les facilite anticipar, resistir o recuperarse de situaciones críticas.

Palabras clave: vulnerabilidad social; indicadores socioeconómicos; valor índice medio.

\begin{abstract}
This paper reveals the socioeconomic vulnerability factors of Acapulco, Guerrero (Mexico). The social vulnerability is a state of human inability manifested collectively and whose genesis is multifactorial. The main objective of this paper is to expose social vulnerability as a neuralgic territorial characteristic to formulate prevention and mitigation criteria, and to assess the potential impact and the occurrence of events that generate adverse consequences. Therefore, conceptual foundations are used to describe and to identify the socio-territorial differences of this Mexican city. These were based on the average index value, which gave guidelines to evaluate the heterogeneous access to a set of assets and structure of opportunities that determine the levels of social vulnerability of the population. Based on this, it was found that most of the acapulqueños lack socioeconomic conditions to anticipate, resist or recover from critical situations.
\end{abstract}

Key words: social vulnerability; socioeconomic indicators; average index value.

Recibido el 14 de noviembre de 2016, aceptado el 13 de abril de 2017.

Consejo Nacional de Ciencias y Tecnología. México. Calle 16 de septiembre núm. 42, Barrio de San Mateo, Chilpancingo, Guerrero, México. c.p.39000. Email: ngarciaca@conacyt.mx. 


\section{Introducción}

Los resultados que se exponen forman parte del proyecto de investigación Gestión multidisciplinaria del riesgo ante fenómenos sísmicos e hidrometeorológicos en Guerrero. En este artículo se aborda la vulnerabilidad social como característica indispensable para la gestión territorial, ya que ésta tiene en cuenta la localización y distribución espacial de los elementos que influyen en el desarrollo y bienestar de la población (Cabrales, 2006). En ese orden de ideas, se concibe que los factores socioeconómicos de vulnerabilidad muestran el acceso diferenciado a un conjunto de activos y estructura de oportunidades que tienen los habitantes de un territorio concreto, lo que les confiere maneras distintas de gestionar el riesgo ante eventos adversos de origen natural o antrópico.

La evaluación de los factores socioeconómicos de vulnerabilidad de los acapulqueños es relevante, ya que éstos habitan en un segmento del territorio nacional expuesto, de manera frecuente a fenómenos sísmicos e hidrometeorológicos, como en septiembre de 2013 con el huracán Ingrid y la tormenta tropical Manuel. Además, Acapulco es uno de los principales destinos turísticos de México; desde la segunda mitad del siglo XX, el turismo ha sido vital no sólo para la dinámica productiva acapulqueña, sino para el territorio guerrerense. Esto lo ha posicionado como la ciudad con mayor importancia económica y demográfica de Guerrero (García \& Sánchez, 2012).

En la literatura especializada se sugiere que el concepto de vulnerabilidad social permite abordar los efectos adversos de fenómenos naturales o antrópicos, desde una perspectiva crítica que subraya las disparidades sociales y económicas de la población, las cuales motivan que ciertos segmentos de ésta resulten más afectados que otros. En ese orden de ideas, las investigaciones sobre el estado de Guerrero, o parte de éste, son escasas; la mayoría de las aportaciones exploran, principalmente, las características de los fenómenos naturales que han ocurrido en la entidad, muestra de esto son los textos de Ramírez \& Lugo-Hubp (2000); Ramírez (2011); Clemente et al. (2012).

La exploración de las diferencias socioeconómicas de un espacio suele ser descriptiva, a menos que se realice desde un enfoque tipológico que separe y clasifique áreas en tipos geográficos con atributos espaciales concretos (Propin \& Sánchez, 1998). Se optó por el enfoque tipológico porque sirve para evaluar, desde la perspectiva cuantitativa y cualitativa, la combinación de los atributos territoriales expresados a través de los datos. Por lo tanto, brinda elementos esenciales para agrupar "datos que evidencian unidades territoriales básicas con un comportamiento similar" (Vázquez, 2007: 100).

Debido a ello, surgió el interés por investigar las particularidades socio-territoriales de Acapulco, mediante el enfoque descrito arriba y difundirlo como referente básico para el examen integral de los atributos sociales y económicos de un espacio. El análisis de los factores socioeconómicos de vulnerabilidad, así como la elaboración de cartografía que los sintetice, es un aporte substancial a la elucidación del origen y las consecuencias de los desequilibrios territoriales presentes en Guerrero. Además, este tipo de investigación es útil para el diseño posterior de criterios de ordenamiento.

Con base en lo anterior, la investigación tiene como objetivo central establecer una alternativa conceptual-metodológica para formular criterios de ordenamiento territorial en la ciudad de Acapulco. Para alcanzar este propósito, se examina el sustento cognoscitivo relacionado con la vulnerabilidad social, se ponderan los factores socioeconómicos de vulnerabilidad que diferencian internamente a esta ciudad guerrerense y se revelan las áreas prioritarias para una gestión territorial socialmente responsable.

\section{Materiales y métodos}

\section{Área de estudio}

La ciudad de Acapulco se localiza en el municipio homónimo del estado de Guerrero, al suroeste de la República 
Mexicana (figura 1). Este asentamiento guerrerense está en la subprovincia fisiográfica denominada Costas del Sur, la cual ocupa la franja territorial adyacente al Océano Pacífico. La llanura costera tiene un ancho que oscila entre los 10 y 25 kilómetros. Sin embargo, en algunas partes la amplitud es menor debido a la incursión de la Sierra Madre del Sur sobre la costa. En algunas laderas de esa forma orográfica, la presencia humana es importante debido a la expansión de la mancha urbana, acaecida en los últimos decenios.

A partir de los años cincuenta, el puerto de Acapulco se erigió como el lugar para vacacionar en México. Eso se debió al mejoramiento de las carreteras que articulaban al puerto con la capital del país, así como a la apertura de un aeropuerto internacional en aquella localidad, en 1964. Éste facilitó el arribo de jets que transportaban una mayor cantidad de personas, además las aerolíneas internacionales empezaron a ofrecer vuelos directos y con costos bajos para aquellos que viajaban en grupo (García \& Sánchez, 2012).

Durante los años setenta, el flujo de turistas que arribó a Acapulco se incrementó considerablemente: en 1970 recibió 1.003 .800 personas y 2.458 .000 , en 1978 . Tal aumento se vio reflejado en la proliferación de la oferta hotelera. Las primeras cadenas de hospedaje que inauguraron una propiedad fueron Hyatt, Marriot, Western, Pierre, Sheraton y Americana Hotels (filial de American Airlines).

El aumento en el número de hoteles e infraestructura indispensable para desempeñar las actividades turísticas transformó el paisaje en la localidad

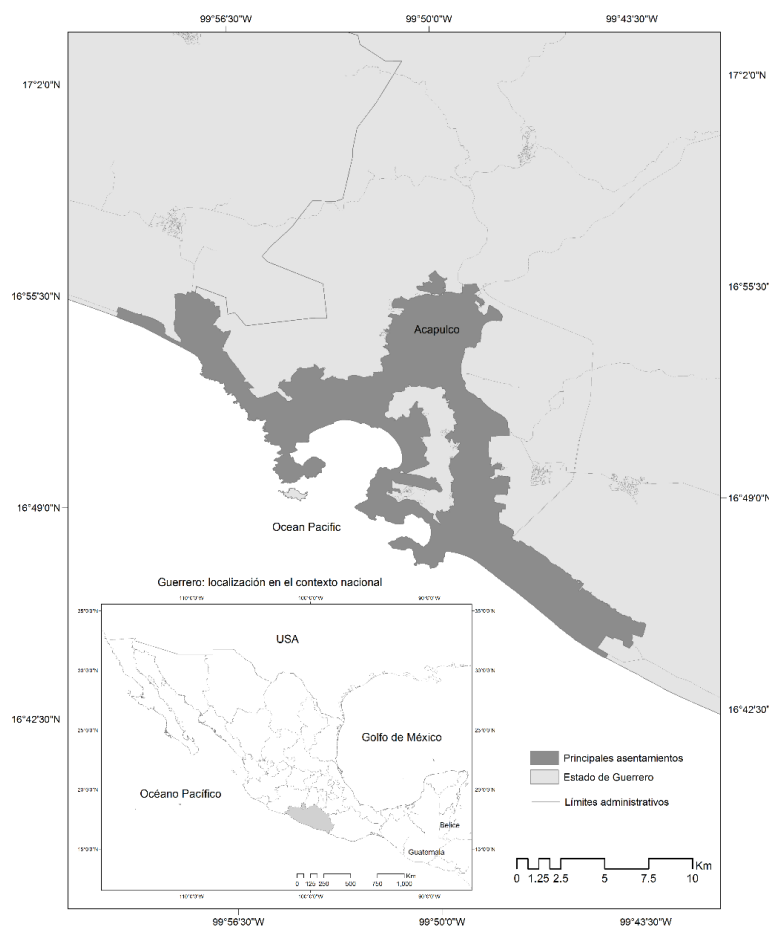

Figura 1. Acapulco: localización del área de estudio.

Fuente: Elaborado con base en Instituto Nacional de Estadística y Geografía (INEGI). (2016)

Figure 1. Acapulco: location of the study area.

Source : Based on Instituto Nacional de Estadística y Geografía (INEGI). (2016) 
guerrerense. Entre 1950 y 1980 , la ciudad creció de 1.250 a 2.726 hectáreas. Se expandió sobre la zona de pie de monte y áreas montañosas (con altitud superior a 225 m.s.n.m.) alrededor de la bahía, así como al noroeste de la localidad.

Debido a la alta demanda de terrenos situados en el área del litoral, las cadenas hoteleras transnacionales optaron por erigir inmuebles de diez o más niveles, los cuales albergaban más de cien habitaciones que ofrecían alojamiento acorde al estilo y confort que demandaba los visitantes, principalmente estadounidenses (Valenzuela \& Coll-Hurtado, 2009).

La etapa de mayor prestigio internacional que vivió Acapulco durante el decenio de los setentas se modificó al inicio de los ochentas, debido a problemas ambientales, sociales y urbanos. En 1980, se registró una reducción mayor al $40 \%$ del total de visitantes extranjeros que tuvo en 1978. El descenso continuó los dos años siguientes y en lo sucesivo, el flujo de turistas presentó un incremento lento con retrocesos mínimos.

No obstante, la disminución de visitantes extranjeros ha sido constante hasta llegar al $1.5 \%$ del total de vacacionistas. A diferencia de lo ocurrido en decenios anteriores, ha tomado relevancia la llegada de turistas nacionales pertenecientes a los estratos socioeconómicos medio y bajo. Esto ha dado pauta a la multiplicación de pequeños y medianos negocios con propietarios locales y/o trabajadores independientes (García \& Sánchez, 2012).

Acapulco ha ejercido atracción de capitales nacionales y extranjeros. Un hecho que ha repercutido en la formación y consolidación de grupos de empresarios que, al amparo de la clase política local y federal, se han apropiado de considerables extensiones territoriales para desarrollar proyectos inmobiliarios, así como de la dotación preferente de infraestructura urbana o de la publicidad indispensable para promover la actividad turística acapulqueña.

El dinamismo de ésta ha propiciado el desarrollo y expansión de su oferta turística. En 1998, Acapulco contó con una inversión de 1.209 millones de dólares en diversos proyectos turísticos. De acuerdo con la Secretaría de Turismo, esto se debe a que los inversionistas nacionales tienen predilección por los destinos de playa consolidados del país (Valenzuela \& CollHurtado, 2009).

Durante el decenio anterior, éstos recibieron 11.607 millones de dólares ( $48 \%$ del total nacional). En dicho período, el estado de Guerrero atrajo la mayor parte de ese monto, esto es 2.625 millones de dólares $(22.6 \%$ del total $)$. De esa inversión, Acapulco concentró el 89.0\%, Ixtapa-Zihuatanejo $6.5 \%$ y la región Norte 4.5\% (García \& Sánchez, 2012).

Con base en el censo 2010 del Instituto Nacional de Estadística y Geografía (INEGI), Acapulco es el centro urbano más grande del estado de Guerrero (673.479 personas). Es uno de los destinos del litoral mexicano que capta más visitantes de origen nacional. En este asentamiento se estima que la población ocupada asciende a 284.165 personas $(87.8 \%$ de la población ocupada municipal) (INEGI 2016).

Sin embargo, la actividad turística también ha impactado de manera negativa en el entorno físico-geográfico; entre las consecuencias más notorias están la fragmentación y destrucción de hábitats distintos, así como la pérdida de biodiversidad existente en éstos; la introducción de especies exóticas; la modificación de la superficie edáfica y del relieve de las áreas donde tiene lugar la construcción de infraestructura turística; el alto consumo y desperdicio de agua; acumulación de residuos sólidos y la contaminación potencial de los recursos hídricos que alteran la calidad de las aguas subterráneas, lagunas y zonas de arrecifes.

Lo anterior no sólo hace evidente una serie de conductas que no tienen en cuenta la capacidad de carga o asimilación del entorno físico-geográfico, sino también la incompatibilidad frecuente de los proyectos turísticos con las tendencias socioeconómicas locales, ya que suele 
ocasionar la pérdida de recursos naturales o la desposesión de éstos. Además, este proceso se basa en la desestructuración territorial de las comunidades involucradas y la imposición de otras lógicas de ocupación y articulación de sus espacios (Cañada, 2010; Secretaría de Medioambiente y Recursos Naturales, 2000).

La ciudad de Acapulco refleja las formas de reproducción del capital, las políticas de Estado, así como las contradicciones sociales derivadas de ambas. Este asentamiento urbano se ha estructurado con base en la lógica del capital y las distintas formas de acumulación de éste. La ciudad creció físicamente debido al incremento significativo que registró el número de habitantes. En términos generales, esto es resultado de la consolidación del turismo como eje de la dinámica económica y el arribo de personas que se desplazan desde las regiones más pobres de Guerrero, en búsqueda de empleo, lo que ha incentivado el crecimiento de barrios marginales en los que residen personas cuyas vidas se caracterizan por la pobreza y pocas oportunidades para modificar tal condición. Desafortunadamente, tal proceso ha tenido lugar sobre las laderas de las montañas que circundan a Acapulco, en consecuencia, los terrenos presentan pendientes pronunciadas e inestabilidad geológica considerable. En esos espacios ha proliferado el establecimiento de población con escasos recursos económicos, debido a los costos elevados de la vivienda y la falta de predios en otras partes céntricas de la ciudad. Las familias suelen obtener, en ocasiones mediante invasión, terrenos no aptos para el desarrollo habitacional y con frecuencia, tienen acceso limitado a servicios públicos (energía eléctrica, agua potable, drenaje).

En este contexto, el objetivo de la presente investigación es establecer una alternativa conceptual-metodológica para formular criterios de ordenamiento territorial en la ciudad de Acapulco. Con este fin, se examinan los aspectos siguientes: 1) el sustento cognoscitivo relacionado con la vulnerabilidad social, 2) se ponderan los factores socioeconómicos de vulnerabilidad que diferencian internamente a esta ciudad guerrerense y 3) se revelan las áreas prioritarias para una gestión territorial socialmente responsable.

\section{Metodología}

El concepto de vulnerabilidad alude a la inseguridad e indefensión que perjudica a un ser humano, a una familia o a un grupo de personas más grande, lo que suele colocarlos por debajo del nivel de bienestar mínimo. Esta condición es multifactorial; sin embargo, suele ser producto, principalmente, de los aspectos siguientes: 1) la probabilidad de que suceda un evento con efectos adversos, 2) la poca o nula respuesta frente a tal contingencia, 3) la falta de mecanismos de defensa apropiados y 4) la inhabilidad para adaptarse a contingencias que entrañe efectos adversos (Centro Latinoamericano y Caribeño de Demografía, 2002; Hufschmidt, 2011; Cecchini, Espíndola, Filgueira, Hernández, \& Martínez, 2012; Cai, 2017; Paveglio \& Edgeley, 2017).

La vulnerabilidad social suele definirse como un estado de incapacidad humana que se manifiesta a nivel individual o colectivo. Es producto tanto de eventos que ocasionan dificultades como de aquellas características socio-territoriales que dificultan la subsistencia, impiden el acceso a mayores niveles de bienestar o favorecen su reducción, imposibilitan el ejercicio de derechos humanos y la consolidación de proyectos personales o colectivos (Kaztman, 2000; Golovanevsky, 2007; Adamo, 2012; Galassi \& González, 2012; Lin, Lee, \& Lin, 2017).

El análisis de la vulnerabilidad social demanda tener en cuenta los factores que agudizan las dificultades enfrentadas por una población concreta, por ejemplo, 1) la exposición a eventos físicos o antrópicos dañinos, 2) los atributos básicos de la población (edad, género, nivel educativo, condición étnica, nivel socioeconómico) y 3) las peculiaridades del modelo económico actual (deterioro del mercado laboral, la volatilidad de los ingresos económicos, la distribución desigual de los activos y la condición endeble adquirida por el Estado) 
(Busso, 2005; Centro Latinoamericano y Caribeño de Demografía, 2002).

Otro aspecto medular de la vulnerabilidad social es su carácter coyuntural, ya que si bien existe una propensión estructural básica (incapacidad humana que se manifiesta a nivel individual o colectivo), esta suele adquirir niveles distintos cuando surge un evento que impacta de manera desfavorable; "de acuerdo con el transcurso de la crisis y con el encadenamiento de los procesos que le acompañan y sus consecuencias perdurables, y de acuerdo con la capacidad de resistencia y de superación de los afectados" (Coy, 2010: 21).

En consecuencia, la vulnerabilidad social tiene variaciones espacio-temporales ocasionadas por la heterogeneidad de los eventos y demás factores que inciden en su manifestación (Adamo, 2012). Desde esa óptica, el impacto diferenciado de un evento dañino refleja rasgos esenciales de interacción, organización y del modelo de estratificación social que regulan la capacidad de anticipar, lidiar, resistir o recuperarse de situaciones críticas (Coy, 2010; Ruíz, 2012; González, Ortecho \& Molinatti, 2013). En ese orden de ideas, la vulnerabilidad social es mayor cuando los activos y la estructura de oportunidades son insuficientes (Filgueira, 2005; Eraso, 2008; Juárez et al., 2014).

Ruíz (2012), en su investigación sobre vulnerabilidad social frente a desastres naturales, expresan que las sociedades desarrolladas y estructuradas tienen mayor capacidad de responder a los desastres, son más resilientes tanto en la fase de emergencia como en la fase de recuperación. Las causas son variadas: por la calidad de sus construcciones e infraestructuras, por su información sobre la catástrofe y de cómo responder frente a la misma, por sus sistemas de alerta temprana, por su capacidad de reacción y movilización, por su estructura organizativa, por los sistemas de gestión de la emergencia, y sobre todo, por su capacidad económica que las permite recuperarse.

Lo anterior ha sido confirmado en investigaciones que sustentan que los factores socioeconómicos de vulnerabilidad son mayores cuando los activos y la estructura de oportunidades son insuficientes (Filgueira, 2005). En consecuencia, su análisis suele relacionarse con el estudio, por ejemplo, de la pobreza, falta de instrucción formal y desprotección social.

Una manera de medir la vulnerabilidad social es mediante la construcción de indicadores con información obtenida de censos. Éstos permiten evaluar, sobre todo, los activos y la estructura de oportunidades que favorecen o dificultan el desempeño de las personas en su contexto social. Asimismo, los censos dan pauta para elaborar caracterizaciones territoriales como las expuestas por Stern (2004); Busso (2005); Alfageme \& Carrasco (2010); Lampis (2010); Arcos et al. (2011); Morales, Rodríguez \& Sánchez, (2013); Álvarez \& Tuñón (2016). En estos trabajos se identifican las principales características estructurales que acentúan la vulnerabilidad social de sectores distintos de población. Esencialmente, se trata de atributos socioeconómicos ocasionados por la acumulación disímil de capacidades y bienes de capital (Cutter, Boruff, \& Shirley, 2003; Sánchez \& Egea, 2011; Sales, 2012).

Al respecto, se considera esenciales los aspectos siguientes: 1) los recursos materiales y simbólicos que favorecen o dificultan el desempeño de las personas en su contexto social (capital financiero, capital humano, experiencia laboral, nivel educativo, composición y atributos de la familia, capital social, participación en redes y capital físico, entre otros); 2) la estructura de oportunidades (determinadas por el mercado, el Estado y la sociedad), las cuales varían debido a las crisis o el crecimiento económico, las transformaciones de la estructura productiva, los cambios tecnológicos y las diversas políticas públicas; y 3) instituciones y relaciones sociales que las personas desarrollan como acción colectiva (familia, sindicatos, empresas, movimientos sociales, partidos políticos) (Pizarro, 2001; Busso, 2005; Feito, 2007; Filgueira, 2005; Golovanevsky, 2007; Birkmann, \& Fernando, 2008; León 2011). 


\section{Procedimiento}

En esta sección se explica cómo se clasificaron las unidades territoriales que integran a la ciudad de Acapulco, con base en el tratamiento metodológico de los indicadores socioeconómicos seleccionados para medir los factores socioeconómicos de vulnerabilidad existente en esta ciudad mexicana. Se empleó información generada por el Instituto Nacional de Estadística y Geografía y el Consejo Nacional de Población (CONAPO).

Para garantizar un examen con detalle territorial significativo, se utilizó los datos por Área Geoestadística Básica urbana (AGEB). Esto es un área geográfica ocupada por manzanas delimitadas por calles, avenidas o cualquier rasgo de fácil identificación en el terreno y cuyo uso de suelo es habitacional, industrial, de servicios o comercial, las cuales sólo son asignadas al interior de las zonas con población mayor o igual a 2.500 habitantes y en las cabeceras municipales (Consejo Nacional de Evaluación de la Política de Desarrollo Social, 2016)*.

El manejo estadístico de estos datos oficiales está regulado por el método del valor índice medio. Éste se utilizó para agrupar unidades espaciales, con base en la repetividad, homogeneidad y jerarquía territorial de éstas. Dicha metodología es relevante para todo análisis tipológico que gravite en torno a la síntesis de los atributos del hecho, fenómeno o proceso en cuestión, ya que facilita la conformación de grupos o tipos discretos que, en lo fundamental, constituyen instrumentos útiles para examinar integralmente el comportamiento de las variables o indicadores que se compendian, de acuerdo al marco teórico-conceptual de referencia (Sánchez, 2000).

En este trabajo se utilizaron indicadores para ponderar los factores socioeconómicos de vulnerabilidad porque se les considera herramientas fundamentales para evaluar, dar seguimiento y predecir tendencias de la situación de un país, estado, municipio o ciudad; por ejemplo, en lo referente a su economía, sociedad, desarrollo humano, etc., así como para valorar el desempeño institucional relacionado con el logro de metas y objetivos establecidos en cada uno de los ámbitos de acción de los programas de gobierno.

En ese orden de ideas, los indicadores utilizados se seleccionaron con base en tres criterios fundamentales; 1) el marco conceptual que orienta la investigación, 2) las particularidades socio-territoriales que refleja cada indicador, lo cual es más importante que la cantidad total de indicadores y 3) la disponibilidad de información estadística oficial (Sánchez, 2000; Propin, 2003; García De León, 2006). En esta ocasión, se emplearon los indicadores siguientes: concentración territorial de población (CTP), tasa de desocupación (TD), relación de dependencia (RD), porcentaje de hogares encabezados por mujeres (HJF) y el índice de marginación urbana (IMU); a continuación se describe cada uno de ellos.

Concentración territorial de la población: Denota la proporción de personas asentadas en una AGEB, respecto al total de la ciudad. Se adoptó debido a que la lectura conjunta de este indicador con los otros cuatro, descritos en este apartado metodológico, permite conocer, de manera sencilla, el porcentaje de población que reportan características socioeconómicas específicas que inciden en la manifestación de vulnerabilidad social frente a posibles eventos adversos de origen natural o humano.

Tasa de desocupación: Muestra la relación entre la población desocupada y la población económicamente activa de cada unidad de análisis. Permite vislumbrar las dificultades del sistema económico para absorber la mano de obra disponible en un espacio;

* Las calificaciones $1,2,3,4,5$ son relativamente arbitrarias. Únicamente son indicativas de la distancia de la variable original, de una unidad territorial, con respecto al valor de la media aritmética. Esto da pauta para ponderar esos valores originales, ya que podrían darse los valores 1, 2 y 3 a los casos bajos y medios, pero asignar 6 (en lugar de 4) a los casos superiores a la media y 10 (en lugar de 5) a los casos muy superiores a la media, para destacar sin duda (aunque rara vez hace falta sobredimensionar esta ponderación) aquellos muy elevados (García De León, 2006). 
esto, dado que los ingresos laborales son la principal fuente de recursos económicos en los hogares, resulta elemental para ponderar la vulnerabilidad económica de la población (Ministerio de Ambiente y Desarrollo Sustentable, 2016) ${ }^{1}$

Relación de dependencia: Alude al porcentaje de personas dependientes (menores de 15 años y de 65 años y más), respecto a la población ocupada en cada AGEB. Cuanto mayor es el valor que ostenta este indicador, en un espacio determinado, resulta más amplia la proporción de habitantes que debe mantener el segmento demográfico que labora y por lo tanto, suele aumentar la vulnerabilidad social (Ministerio de Ambiente y Desarrollo Sustentable, 2016). Además, valorar la cantidad de población infantil y de la tercera edad es fundamental porque ambos grupos suelen presentar vulnerabilidad notable ${ }^{2}$.

Porcentaje de hogares con jefatura femenina: Revela la proporción de hogares encabezados por mujeres, en relación al total de hogares cuantificados en el espacio evaluado. Investigaciones nacionales e internacionales han expuesto que este tipo de hogares son más vulnerables, por ejemplo, debido a la mayor carga de trabajo a la que se enfrentan las madres solteras y a la falta de conformación de capital común con una pareja (Sales, 2012).
El índice de marginación urbana muestra el impacto que tiene: 1) la escasa instrucción formal de la población, 2) los servicios de salud deficientes, 3) la residencia en viviendas inadecuadas y 4) la falta de bienes en éstas. De esta manera, con base en el análisis simultáneo de los cinco parámetros planteados en esta investigación, se obtuvo una imagen de los factores socioeconómicos de vulnerabilidad que presenta la ciudad de Acapulco. Cabe destacar que el procedimiento para calcular cada uno de ellos, está descrito en el trabajo de García \& Villerías (2016).

Asimismo, la investigación de García De León (2006) puntualiza como calcular el valor índice medio, los pasos sustanciales son: 1) construir una matriz con los parámetros para cada AGEB, 2) cálculo de la media aritmética y la desviación típica del conjunto de datos asociado con cada indicador (tabla 1), 3 ) tipificación de los valores originales de la matriz (se resta la media aritmética a los valores de los indicadores y el resultado se divide entre la desviación típica (tabla 2), 4) calificación de los valores tipificados con base en los rangos calculados para el conjunto de datos (tabla 3 y 4) (figuras 2 a 6), 5) cálculo del valor índice medio (resulta del promedio de los cinco valores tipificados que caracterizan a cada AGEB) (tabla 5) y 6) calificación cualitativa del valor índice medio con base en los rangos calculados

1 Si bien, la tasa de desocupación puede ocultar realidades tales como: el porcentaje de empleo informal importante, un desempleo relativamente bajo inducido en una época del año donde incrementa el empleo temporal, porcentaje de subempleo importante, entre otras realidades; este indicador se seleccionó ya que no se cuenta con información sobre el ingreso de la población ocupada por AGEB. Además, el índice de marginación urbana, utilizado en esta investigación, toma en cuenta indicadores sobre la capacidad del ingreso de los hogares. De esta manera, la tasa de desocupación afina los escenarios revelados por este último indicador.

2 Este indicador requiere una lectura prudente, puesto que existen poblaciones en donde hay un porcentaje importante de jóvenes menores de 15 años que trabajan, así como población adulta mayor que labora (incluso siendo jubilada de un primer trabajo). También puede ocurrir que adultos (hipotéticamente activos) no trabajen y dependan de otros adultos o incluso de otros grupos como los jóvenes. Sin embargo, se optó por la relación de dependencia porque permite ponderar, de manera conjunta, la presencia de dos sectores de la población que presentan mayor vulnerabilidad (niños y ancianos). 
Tabla 1

Ejemplo del comportamiento cuantitativo de los indicadores seleccionados. Fuente: Elaborado con base en INEGI (2016)

Table 1

Example of quantitative behavior of the selected indicators. Source: Based on INEGI, 2016

\begin{tabular}{lccccc}
\hline AGEB & CTP & \multicolumn{1}{c}{ TD } & RD & HJF & IMU \\
\cline { 2 - 6 } 0034 & 0,601 & 5,201 & 81,336 & 40,835 & 0,065 \\
0049 & 0,635 & 1,408 & 76,878 & 42,773 & $-0,037$ \\
0091 & 0,457 & 2,012 & 59,138 & 25,602 & $-0,738$ \\
0104 & 0,341 & 5,059 & 91,270 & 30,328 & $-0,541$ \\
---- & ----- & ----- & ----- & ---- & ----- \\
---- & ----- & ------- & -- \\
6419 & 0,023 & 3,529 & 63,415 & 28,333 & $-1,293$ \\
6438 & 0,283 & 4,459 & 79,347 & 24,573 & $-0,403$ \\
6442 & 0,459 & 3,656 & 83,929 & 23,160 & $-0,552$ \\
Suma* & 100 & $1.928,864$ & $3.8820,904$ & $13.425,441$ & 181,832 \\
Media* & 0,216 & 4,637 & 84,947 & 30,582 & 0,448 \\
Desviación & 0,182 & 2,958 & 21,716 & 7,841 & 1,084 \\
típica* & & & & & \\
\hline
\end{tabular}

Tabla 2

Ejemplo de indicadores tipificados. Fuente: Elaborado con base en la tabla 1. (2016)

Table 2

Example of indicators typified. Source: Based on table 1. (2016)

\begin{tabular}{|c|c|c|c|c|c|}
\hline AGEB & CTP & TD & RD & HJF & IMU \\
\hline 0034 & 2,1210 & 0,1906 & $-0,1663$ & 1,3077 & $-0,3532$ \\
\hline 0049 & 2,3058 & $-1,0912$ & $-0,3716$ & 1,5548 & $-0,4474$ \\
\hline 0091 & 1,3288 & $-0,8872$ & $-1,1885$ & $-0,6351$ & $-1,0939$ \\
\hline 0104 & 0,6903 & 0,1428 & 0,2912 & $-0,0324$ & $-0,9120$ \\
\hline 6419 & $-1,0608$ & $-0,3743$ & $-0,9916$ & $-0,2868$ & $-1,6050$ \\
\hline 6438 & 0,3699 & $-0,0600$ & $-0,2579$ & $-0,7663$ & $-0,7848$ \\
\hline 6442 & 1,3403 & $-0,3315$ & $-0,0469$ & $-0,9465$ & $-0,9218$ \\
\hline
\end{tabular}

* Calculadas con base en el conjunto de datos de todas las AGEB que integran Acapulco.

Nota: las líneas segmentas advierten la existencia de datos de las otras 457 AGEB de la ciudad. 
Tabla 3

Calificaciones para valores tipificados. Fuente: Elaborado con base en la tabla 2. (2016)

Table 3

Ratings for standardized values. Source: Based on table 2. (2016)

\section{Calificación}

1

2

3

4

5

\section{Rango de aplicación}

Menor a $-1,0$

Entre - 1,0 y -0,50

Entre $-0,51$ y 0,50

Entre 0,51 y 1,0

Mayor a 1,0

Nota:

1. Si el valor tipificado resultante es menor de $-1,00$, se le asigna calificación de 1 (la más baja de todas, debido a que corresponde a un valor original también muy bajo).

2. Entre $-0,50$ y $-1,0$, calificación de 2 (corresponde también a un valor original bajo, pero ya no tanto).

3. De $-0,50$ a 0,50 va la calificación de 3 (Por un valor original ya más cercano a la media, donde la teoría de la probabilidad asume que se tendrán $68 \%$ del total de casos).

4. De 0,5 a 1,0 califica con 4 . Se trata de un valor original que supera la media del conjunto.

5. Mayor de 1,0 obtendrá 5. El valor más alto de todos ya que supera por mucho la media.

Tabla 4

Ejemplo de la cualificación de los indicadores utilizados. Fuente: Elaborado con base en la tabla 2 y 3. (2016)

Table 4

Example of the qualification of the indicators used. Source: Based on table 2 and 3. (2016)

\begin{tabular}{|c|c|c|c|c|c|}
\hline AGEB & CTP & TD & RD & HJF & IMU \\
\hline 0034 & 5 & 3 & 3 & 5 & 3 \\
\hline 0049 & 5 & 1 & 3 & 5 & 3 \\
\hline 0091 & 5 & 2 & 1 & 2 & 1 \\
\hline 0104 & 4 & 3 & 3 & 3 & 2 \\
\hline 6419 & 1 & 3 & 2 & 3 & 1 \\
\hline 6438 & 3 & 3 & 3 & 2 & 2 \\
\hline 6442 & 5 & 3 & 3 & 2 & 2 \\
\hline
\end{tabular}

Nota: las líneas advierten la existencia de datos de las otras 457 AGEB de la ciudad. 


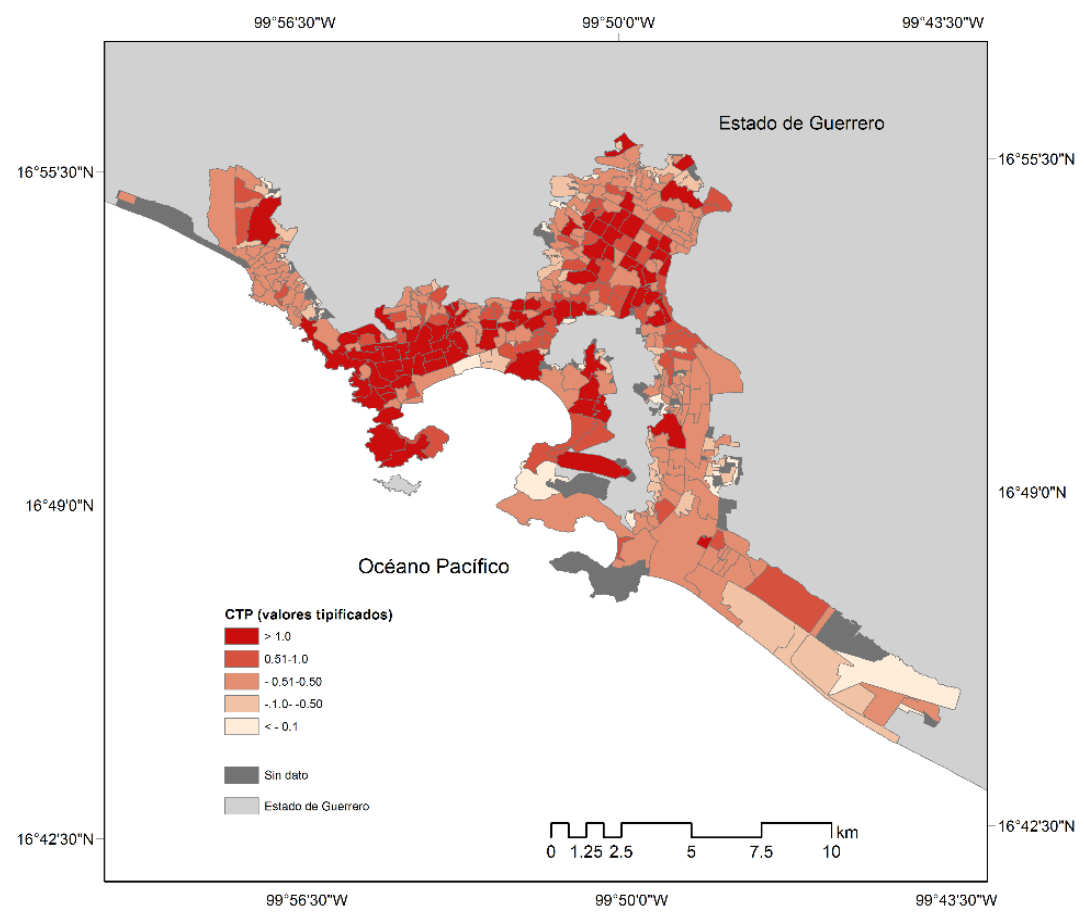

Figura 2. Acapulco: concentración territorial de población. Fuente: Elaborado con base en INEGI. (2016)

Figure 2. Acapulco: territorial concentration of population. Source: Based on INEGI. (2016)

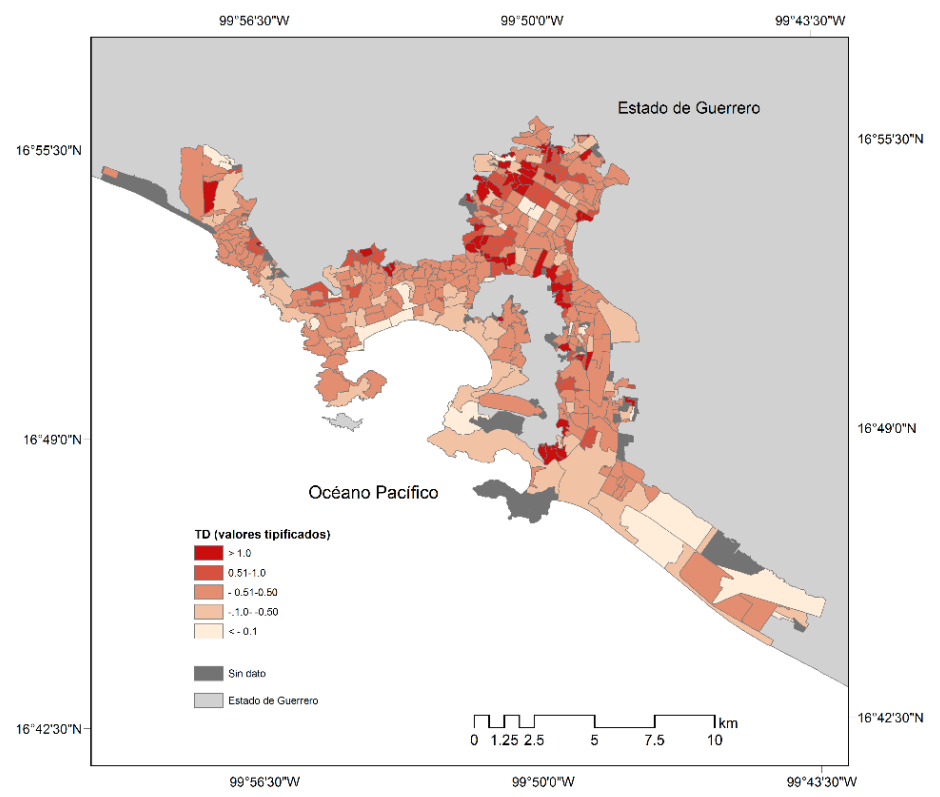

Figura 3. Acapulco: tasa de desocupación. Fuente: Elaborado con base en INEGI. (2016)

Figure 3. Acapulco: unemployment rate. Source: Based on INEGI. (2016) 


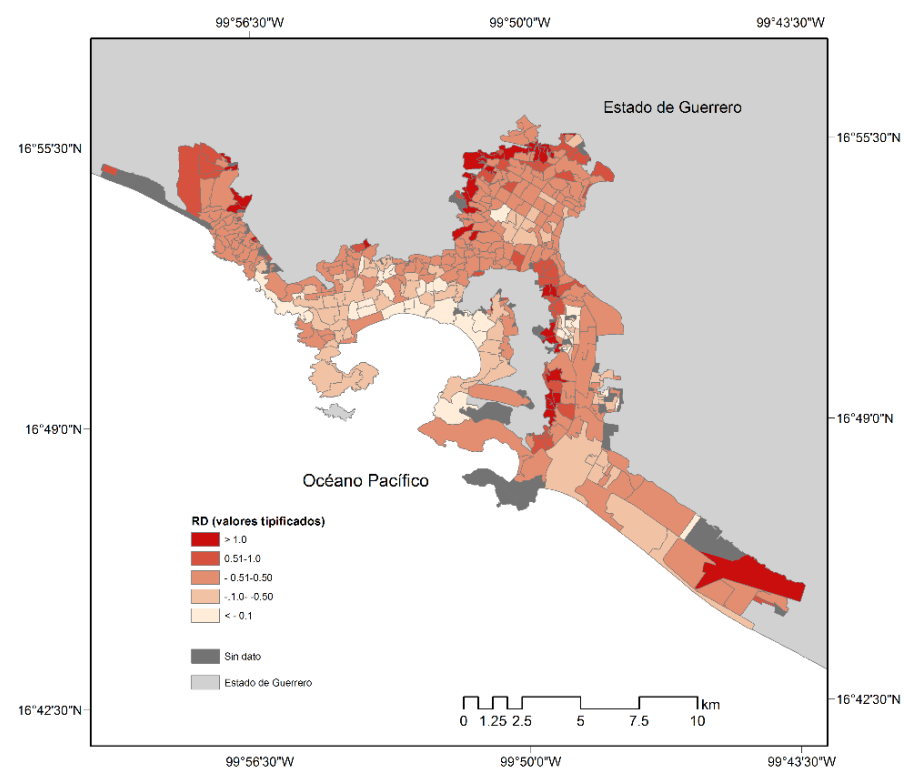

Figura 4. Acapulco: tasa de dependencia. Fuente: Elaborado con base en INEGI. (2016)

Figure 4. Acapulco: dependency ratio. Source: Based on INEGI. (2016)

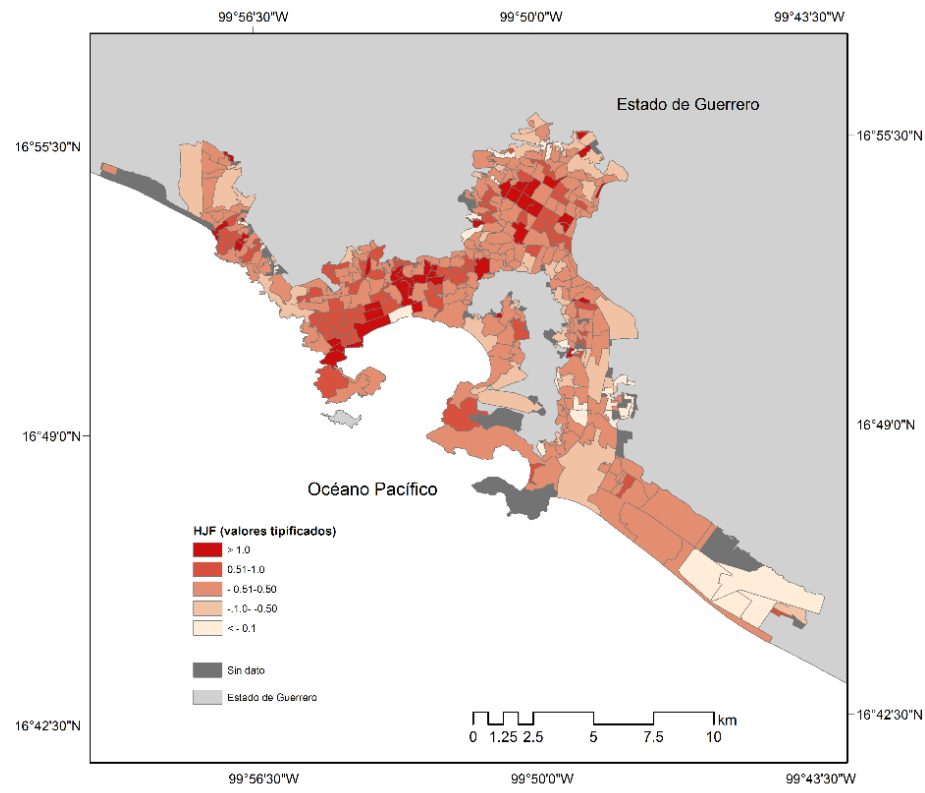

Figura 5. Acapulco: porcentaje de hogares encabezados por mujeres. Fuente: Elaborado con base en INEGI. (2016)

Figure 5. Acapulco: female-headed households. Source: Based on INEGI. (2016) 


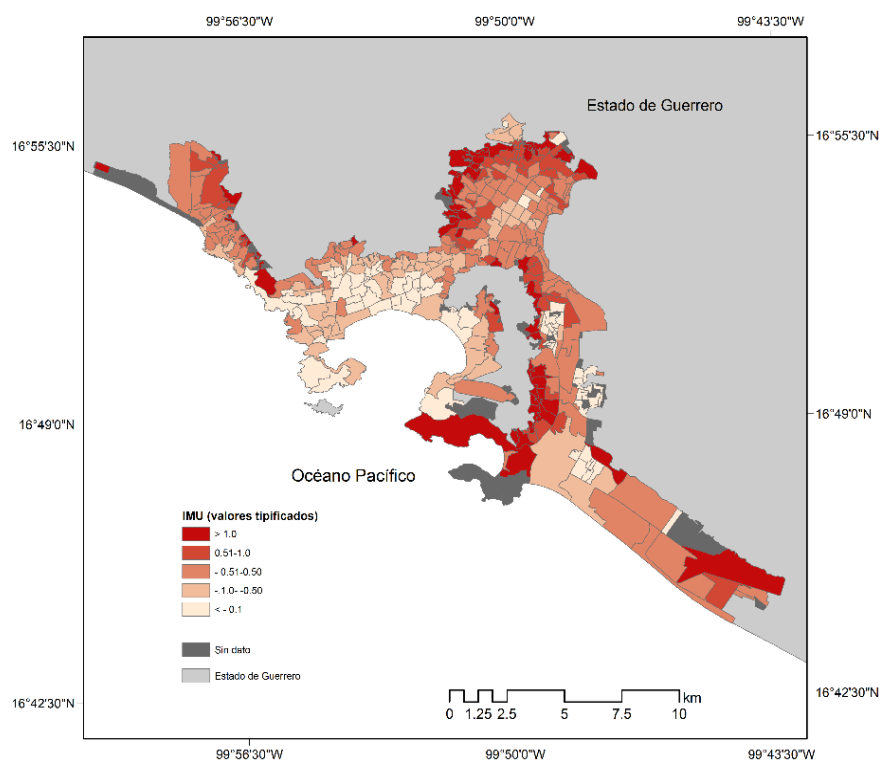

Figura 6. Acapulco: índice de marginación urbana. Fuente: Elaborado con base en INEGI. (2016)

Figure 6. Acapulco: urban marginalization index. Source: Based on INEGI. (2016)

Tabla 5

Ejemplo del cálculo del valor índice medio. Fuente: Elaborado con base en la tabla 4. (2016)

Table 5

Example of calculation of the mean index value. Source: Based on table 4. (2016)

\begin{tabular}{|c|c|c|c|c|c|c|}
\hline AGEB & CTP & TD & RD & HJF & IMU & VIM \\
\hline 0034 & 5 & 3 & 3 & 5 & 3 & 3,8 \\
\hline 0049 & 5 & 1 & 3 & 5 & 3 & 3,4 \\
\hline 0091 & 5 & 2 & 1 & 2 & 1 & 2,2 \\
\hline 0104 & 4 & 3 & 3 & 3 & 2 & 3,0 \\
\hline 6419 & 1 & 3 & 2 & 3 & 1 & 2,0 \\
\hline 6438 & 3 & 3 & 3 & 2 & 2 & 2,6 \\
\hline 6442 & 5 & 3 & 3 & 2 & 2 & 3,0 \\
\hline
\end{tabular}

Nota: las líneas advierten la existencia de datos de las otras 457 AGEB de la ciudad. 
Tabla 6

Calificaciones para el VIM. Fuente: Elaborado con base en la tabla 5. (2016).

Table 6

Ratings for the MIV. Source: Based on table 5. (2016)

\begin{tabular}{ll}
\hline Calificación & Rango de aplicación \\
\cline { 2 - 2 } 1 & Menor a 1,68 \\
2 & Entre 1,69 y 2,36 \\
3 & Entre 2,37 y 3,04 \\
4 & Entre 3,05 y 3,72 \\
5 & Mayor a 3,72 \\
\hline
\end{tabular}

Nota: con el fin de ordenar las AGEB en rangos de clasificación adecuados, se aprovechó el valor del índice medio obtenido. Con base en los valores obtenidos, se observó la posibilidad de formar cinco grandes grupos "naturales". A partir de esa agrupación es posible jerarquizar las AGEB, en rangos con amplitud aceptable, justificados por su similitud con la secuencia que presentan las unidades tipificadas que permiten formar la curva de distribución normal (García De León, 2006)

Tabla 7

Ejemplo de la determinación de la condición de vulnerabilidad social. Fuente: Elaborado con base en la tabla 6. (2016).

Table 7

Example of the determination of the social vulnerability condition. Source Based on table 6. (2016)

\begin{tabular}{|c|c|c|}
\hline AGEB & VIM & Condición \\
\hline 0034 & 3,8 & 5 \\
\hline 0049 & 3,4 & 4 \\
\hline 0091 & 2,2 & 2 \\
\hline 0104 & 3,0 & 3 \\
\hline 6419 & 2,0 & 2 \\
\hline 6438 & 2,6 & 3 \\
\hline 6442 & 3,0 & 3 \\
\hline
\end{tabular}

Nota: las líneas advierten la existencia de datos de las otras 457 AGEB de la ciudad. 


\section{Resultados}

En la ciudad de Acapulco, las condiciones de vulnerabilidad social no siempre se asocian con un conjunto de indicadores socioeconómicos cuyas expresiones cuantitativas sean, únicamente, altas (4) o muy altas (5). Tampoco la condición mínima tiene sólo expresiones muy bajas (1) y bajas (2). Por ejemplo, el comportamiento del porcentaje de hogares encabezados por mujeres registra 3 o más puntos en AGEB con una condición muy baja (1) o baja (2). En contraste, este mismo indicador no supera ese puntaje en más del $60 \%$ de las unidades territoriales que reportan una condición muy alta (5). La tipología que se expone a continuación arroja luz en torno a las condiciones de vulnerabilidad social, con base en el comportamiento global de los indicadores ponderados. Por lo tanto, siempre resulta conveniente revisar el comportamiento individual para detectar las áreas prioritarias en cada estrato revelado.

El método del valor índice medio dio pauta para clasificar los factores socioeconómicos de vulnerabilidad en las condiciones siguientes:

Muy baja. Se detectó en un total de cinco AGEB con la distribución geográfica siguiente: tres se ubican muy próximas alárea costera; es el caso de los fraccionamientos Las Brisas, Condesa y la zona del Parque Papagayo (figura 7). El resto de las AGEB pertenecen a la periferia nororiental de la ciudad de Acapulco; básicamente, se trata de la zona del fraccionamiento Joyas del Marqués II. En términos generales, estas AGEB tienen una condición de vulnerabilidad muy inferior al promedio, no superan 1.6 puntos como valor medio debido al predominio de cifras bajas, en los indicadores ponderados. Únicamente, la tasa de desocupación y el porcentaje de hogares encabezados por mujeres registran valores medios (3) y altos (4), en un par de AGEB. Lamentablemente, en estas cinco circunscripciones viven sólo 741 personas ( $0.1 \%$ del total de habitantes).

Baja: Corresponde a veintiocho AGEB que muestran presencia notable en la costa y en la parte oriental de Acapulco; destaca la Colonia Adolfo López Mateos, Lomas de Mozimba, Magallanes, Granjas del Marqués, El Pueblito del Revolcadero, Fraccionamiento Copacabana, Playa Diamante y El Coloso. Allí el valor índice medio oscila entre 1.8 y 2.0 puntos. Los indicadores con valores sobresalientes son: la concentración territorial de población y el porcentaje de hogares con jefatura femenina; aproximadamente, $50 \%$ de las AGEB evaluadas muestra valores medios (3). Por su parte, la tasa de desocupación exhibe este puntaje en $40 \%$ de las AGEB. $\mathrm{El}$ resto de los indicadores tiene predominio de valores muy bajos (1) y bajos (2). Este conjunto de características se detecta en 28.467 personas $(4.2 \%$ del total de acapulqueños).

Media: Hay ciento cuarenta y nueve AGEB con vulnerabilidad social media $(38.2 \%$ del total). Se encuentran dispersas en el puerto de Acapulco; pero tienen presencia considerable en la sección occidental de éste. En esa parte la ciudad, la dimensión de cada AGEB es menor, comparada con la que tienen en la parte oriental, además aquí hay un número relevante de fraccionamientos habitacionales. El valor índice medio varía entre 2.4 y 3.0 puntos; esto se debe a que cuatro de cinco indicadores ponderados tienen valores medios (3), en alrededor de $50 \%$ de las AGEB. En contraste, el índice de marginación urbana registra sólo 30\% de casos con este puntaje. Esta condición de vulnerabilidad social corresponde a 257.249 personas (38\% de los habitantes de Acapulco).

Alta: Existen ciento cincuenta y nueve AGEB con vulnerabilidad social alta. Esto es recurrente el occidente y norte de Acapulco. En este último sobresalen colonias como Ciudad Renacimiento, Zapata, San José Cacahuatepec y el Fraccionamiento Libertadores. Hay 321.041 acapulqueños con vulnerabilidad social alta (47.7\% de la población de la ciudad). El valor índice medio fluctúa entre 3.2 y 3.6 puntos. Esta vulnerabilidad social alta está asociada con las AGEB que se distinguen por la concentración territorial de población alta (4) y muy alta (5) (47\% de los casos). 


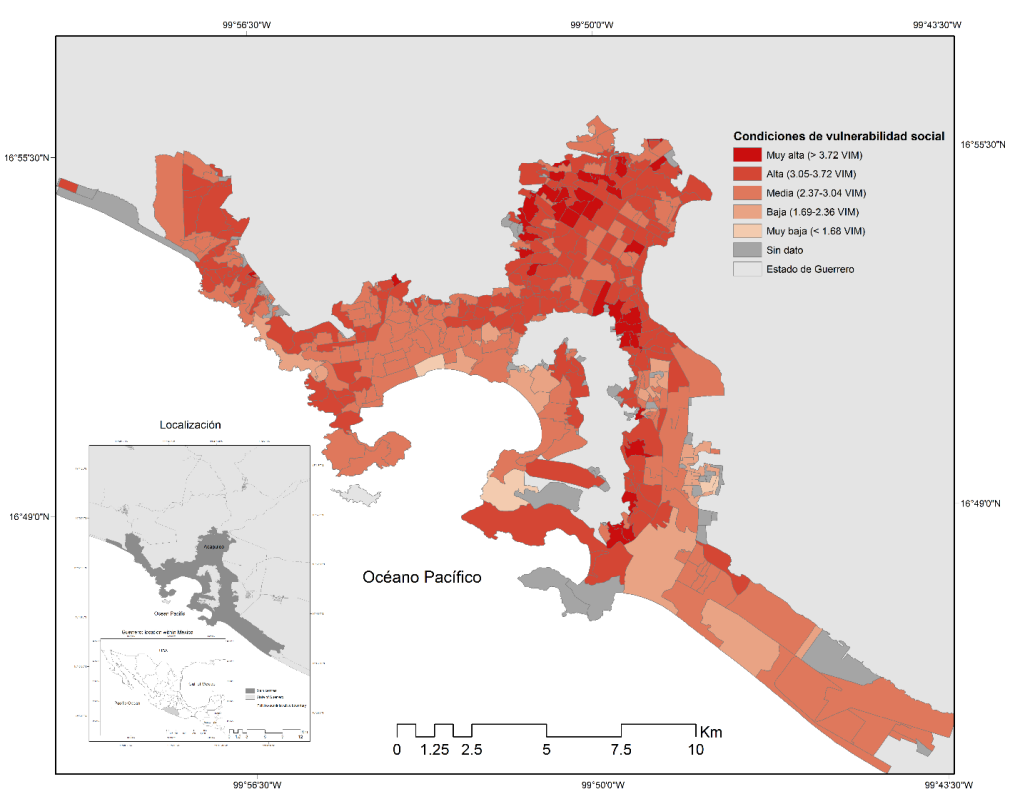

Figura 7. Acapulco: condiciones de vulnerabilidad social. Fuente: Elaborado con base en INEGI (2016)

Figure 7. Acapulco: conditions of social vulnerability. Source: Based on INEGI (2016)

Los indicadores restantes también presentan esos valores; el índice de marginación urbana se comporta de ese modo, en $41 \%$ de las AGEB; la tasa de desocupación, en $33 \%$ de los casos; el porcentaje de hogares encabezados por mujeres, en $28 \%$ de las AGEB; y la relación de dependencia, en $26 \%$ de los casos.

Muy alta: Corresponde a un total de ciento noventa AGEB localizadas, sobre todo, en la parte nororiental de esta ciudad guerrerense. Esta condición corresponde a 55.916 personas, esto es $8.3 \%$ de la población de Acapulco. El valor índice medio oscila entre 3.8 y 4.4 puntos debido a la presencia importante de valores altos (4) y muy altos (5); en particular, la tasa de desocupación y el índice de marginación urbana tienen estos puntajes, en más del $80 \%$ de los casos evaluados. La relación de dependencia se comporta de esa manera en $70 \%$ de las ocasiones. Por otra parte, la concentración territorial de población y el porcentaje de hogares encabezados por mujeres, tuvieron menos relevancia cuantitativa, ya que más del $60 \%$ de los casos no superan los 3 puntos.

\section{Discusión}

La mayor parte de los habitantes de la ciudad de Acapulco presenta factores socioeconómicos de vulnerabilidad significativos. En la sección anterior de esta investigación se exponen hallazgos que dan pauta para advertir como ese rasgo estructural de la población segmenta en niveles distintos a los residentes de una ciudad, lo cual confirma la utilidad de los instrumentos cuantitativos para elaborar caracterizaciones territoriales como la expuesta en este trabajo, o en aquellos desarrollados por especialistas en la materia, tal es el caso de Busso (2005); Pavarini et al. (2009); Alfageme \& Carrasco (2010); Lampis (2010) y Araujo (2015).

En el análisis de los factores socioeconómicos de vulnerabilidad que ostentan los acapulqueños es conveniente acotar los aspectos siguientes:

Desde el decenio de los cincuentas, la actividad turística ha sido estimulada por el gobierno mexicano; asimismo, más tarde, ya con el modelo neoliberal, también 
cobró importancia la inversión extranjera directa relacionada con esta actividad económica. Esto permitió que Acapulco se posicionará como uno de los principales destinos turísticos del país, pero también trajo consigo la imposición de un conjunto de condiciones políticas y económicas que sólo garantizan la obtención de utilidades a los inversionistas.

En consecuencia, se registraron impactos socio-ambientales diversos, sobre todo, en las áreas involucradas, directamente, con la puesta en marcha de proyectos turísticos. La mayoría de los conflictos, ha tenido lugar tras la disputa por espacios dotados de características físicas estratégicas para el desarrollo de los mismos, así como por la incertidumbre socioeconómica de las personas que laboran en este destino de playa; en particular aquellos con instrucción formal mínima, lo que los obliga a desempeñar trabajos informales, sin recibir seguridad social o tener certeza laboral.

Los factores socioeconómicos de vulnerabilidad identificados en Acapulco es resultado del carácter minimizado que tiene el Estado mexicano, lo que ha derivado en la reducción y en lo sucesivo, la falta de políticas sociales. Esto tuvo un efecto directo sobre la polarización social que se observa tanto en los factores socioeconómicos de vulnerabilidad de los habitantes de la ciudad como en la morfología de la misma.

El método del valor índice medio dio pauta para identificar las situaciones que se describen a continuación. Primero, en términos generales, las condiciones de vulnerabilidad social coinciden con el comportamiento socio-territorial registrado en otros espacios del Pacífico mexicano, tal y como se advierte en los trabajos de Juárez, Iñiguez, \& Sánchez (2006), así como en la investigación de Rodríguez, Ruz, \& Hernández (2012). En ambos casos, los autores exponen cómo los centros turísticos muestran una estructura urbana altamente diferenciada; las diferencias espaciales, económicas y sociales son notables, sobre todo entre las áreas destinadas a los visitantes y aquellas en las que reside la población.

Sin embargo, es conveniente acotar que en el caso de Acapulco, los factores socioeconómicos de vulnerabilidad tiene una expresión espacial más heterogénea, si bien las áreas con condición muy baja (1) y baja (2) se asocian con la zona hotelera y los fraccionamientos residenciales para personas con ingresos económicos considerables, no hay una separación clara entre zonas que ostentan distintos factores sociales de vulnerabilidad; las AGEB con condición alta (4) y muy alta (5) se ubican, con frecuencia, cerca de espacios estratégicos para el turismo, lo que advierte la existencia de una periferia interior en esta ciudad guerrerense.

Este asentamiento guerrerense presenta un panorama socio-territorial complejo. Las AGEB con mayor concentración demográfica son también las que muestran mayores factores socioeconómicos de vulnerabilidad; más del $50 \%$ de los acapulqueños supera la condición media. En contraste, menos del 5\% de los habitantes están por debajo de esta categoría; no obstante, el comportamiento individual de algunos indicadores denota la existencia de disparidades en estos espacios privilegiados por la inversión pública y privada.

El turismo ha transformado el paisaje de esta localidad guerrerense, debido a la construcción de hoteles y demás infraestructura para recibir a los visitantes. Paralelamente, desde el decenio de los cincuenta, la expansión del número de habitantes de la ciudad originó que las autoridades, a menudo, regularizarán las colonias que se erigieron mediante la invasión de predios. Por lo tanto, como en otras ciudades mexicanas, la mayoría de las ocasiones, en la gestión urbana acapulqueña no se ha tenido en cuenta la capacidad y aptitud de las áreas sobre la que tuvo lugar la expansión de este asentamiento humano.

Lo anterior permite comprender porque en Acapulco existen muchos espacios sin servicios públicos y además expuestos a los efectos adversos de los 
fenómenos hidrometeorológicos (sobre todo inundaciones y movimientos gravitacionales). Estos últimos se presenta con mayor frecuencia en las laderas de las formaciones orográficas que circundan al asentamiento urbano, lo cual es relevante para el diseño de instrumentos relacionados con la gestión territorial, ya que los resultados de la presente investigación exponen que allí se hallan las AGEB con mayores factores socioeconómicos de vulnerabilidad. En tanto, las áreas que suelen inundarse son recurrentes, incluso en aquellos espacios con vulnerabilidad social mínima, debido a que la gestión local ha dado prioridad al sector inmobiliario con base en un enfoque meramente financiero.

La literatura especializada expone los factores que agudizan las dificultades enfrentadas por una población, frente a fenómenos adversos de origen natural o antrópico, sin embargo, en el caso de Acapulco es necesario que se identifiquen los factores socioeconómicos de vulnerabilidad que le dan un carácter socio-territorial particular a esta ciudad, ante probables eventos desfavorables como los señalados arriba; además del análisis con indicadores como los utilizados en esta investigación, es relevante considerar de qué manera se da la incorporación de este destino turístico en el contexto nacional y global, así como sus implicaciones socioeconómicas para los acapulqueños.

El valor índice medio es una propuesta metodológica novedosa, debido a su utilidad para detectar los contrastes socioeconómicos de la población, con base en información cuantitativa. Desafortunadamente, en la mayoría de los países en desarrollo, las fuentes estadísticas oficiales no poseen variables suficientes para elaborar un análisis integral de la vulnerabilidad social. Pese a ello, representa la alternativa más viable para sustentar estudios con alcance territorial considerable, ya que la recopilación de datos in situ demanda recursos financieros y humanos considerables.

La tipología que se revela en esta investigación podría constituir un soporte para diseñar políticas públicas que tengan como objetivo mejorar las condiciones socioeconómicas de los habitantes de un territorio concreto, lo cual resulta apremiante debido a la escases de instrumentos de ese tipo que caracteriza al contexto neoliberal. Es útil para vislumbrar algunas de las transformaciones asociadas con el modelo económico impuesto en América Latina, sobre todo da pauta para identificar cómo segmentos amplios de la población está indefensa ante eventos adversos (naturales o antrópicos), ya que si bien esta condición afecta con mayor frecuencia a los sectores con menores ingresos, ningún estrato social está exento.

\section{Conclusión}

En la ciudad de Acapulco hay trecientas cuarenta y nueve AGEB prioritarias, ya que éstas muestran condiciones altas (4) y muy altas (5), en cuanto a factores socioeconómicos de vulnerabilidad. Allí vive $56 \%$ de los acapulqueños; de acuerdo con el marco de referencia de esta investigación, éstos no poseen capacidad suficiente para compensar los efectos negativos que pueden generar algunos fenómenos, naturales o antrópicos, sobre su bienestar. En contraste, menos del 5\% de los habitantes de la ciudad tiene capacidad substancial de resiliencia tanto en las fases de emergencia como en la recuperación derivadas de un evento adverso, esto es sólo aquellas personas que viven en las treinta y tres AGEB con condición baja (2) y muy baja (1).

En términos generales, las AGEB con condición baja (2) y muy baja (1) se asocian con la zona hotelera y residencial exclusiva de Acapulco. Las unidades espaciales que registran valores superiores a la condición media son aquellas en las que tienen presencia importante los predios que aún no interesan a las compañías inmobiliarias, sin que necesariamente existan distancias considerables entre esos espacios y las áreas propicias para desarrollar actividades turísticas. No obstante, es en la periferia donde los indicadores ponderados registran valores altos (4) y muy altos (5).

Lo anterior revela la estructura territorial polarizada que existe en esta ciudad del 
estado de Guerrero; la mayor parte de la población local tiene características socioeconómicas que advierten dificultades para subsistir, poseen niveles mínimos de bienestar, no pueden ejercer sus derechos humanos y en consecuencia, no es capaz de consolidar proyectos a nivel personal, familiar o comunal. Por lo tanto, existe una cantidad notable de personas con capacidad escasa para hacer frente a situaciones de emergencia de distinta índole y magnitud.

La tipología revelada, como ocurre con los trabajos de corte geográfico-estadístico que utilizan la división político-administrativa como unidad de análisis, brinda una imagen general de la cobertura espacial de las características socioeconómicas en cada AGEB. Por lo tanto, en investigaciones futuras, será substancial afinar los escenarios descubiertos mediante un examen in situ; a pesar de la cobertura territorial reducida que pueda tener éste y los recursos financieros y humanos que suele demandar la puesta en práctica de técnicas propias del trabajo en campo.

\section{Referencias}

Adamo, S. B. (julio, 2012). Vulnerabilidad social. En Taller nacional sobre desastre, gestión de riesgo y vulnerabilidad: Fortalecimiento de la integración de las ciencias naturales y sociales con los gestores de riesgo. Buenos Aires, Argentina.

Álvarez, G.C. \& Tuñón, E. (2016). Vulnerabilidad social de la población desplazada ambiental por las inundaciones de 2007 en Tabasco (México). Cuadernos de Geografia: Revista Colombiana de Geografia, 25 (1), 123-138. https://doi. org/10.15446/rcdg.v25n1.52591

Araujo, R. (2015). Vulnerabilidad y riesgo en salud: ¿dos conceptos concomitantes? Revista Novedades de Población, 11(21), 89-96.

Arcos, E., Muñoz, L.A., Sánchez, X., Vollrath, A., Latorre, C., Bonatti, C., \& Jauregui, J. (2011). Vulnerabilidad social en mujeres embarazadas de una comuna de la región metropolitana. Revista Médica de Chile, 139(6), 739-747. https://doi. org/10.4067/s0034-98872011000600007

Alfageme, M.A. \& Carrasco, A. (octubre, 2010). Midiendo la vulnerabilidad de los hogares en el Perú. En XXVIII Encuentro de economistas banco central de reserva del Perú. Lima, Perú.

Birkmann, J. \& Fernando, N. (2008). Measuring revealed and emergent vulnerabilities of coastal communities to tsunami in Sri Lanka. Disasters, 32 (1), 82-105. https:// doi.org/10.1111/j.1467-7717.2007.01028.x

Busso, G. (octubre, 2005). Pobreza, exclusión y vulnerabilidad social. Usos, limitaciones y potencialidades para el diseño de politicas de desarrollo $y$ de población. En VIII Jornadas argentinas de estudios de población, Asociación de Estudios de la Población. Buenos Aires, Argentina.

Cabrales, L.F. (2006). Geografía y ordenamiento territorial. D. Hiernaux, A. Lindón (Eds.), Tratado de Geografia Humana (pp. 601-627). Barcelona: Anthropos, UAMIztapalapa.

Cai, Y. (2017). Bonding, bridging, and linking: photovoice for resilience through social capital. Natural Hazards, 87 (2): 1-27. https:// doi.org/10.1007/s11069-017-2913-4

Cañada, E. (2010). Turismo en Centroamérica, nuevo escenario de conflicto social. Managua: AlbaSud.

Cecchini, S., Espíndola, E., Filgueira, F., Hernández, D., \& Martínez, R. (2012). Vulnerabilidad de la estructura social en América Latina: medición y políticas públicas. Realidad, datos y espacio. Revista Internacional de Estadística y Geografia, 3 (2), 32-45.

Centro Latinoamericano y Caribeño de Demografía, CELADE (2002). Capítulo I y V. En CELADE (ed.), Vulnerabilidad 
sociodemográfica: viejos y nuevos riesgos para comunidades, hogares y personas. (pp. 1-69). Brasilia: Publicaciones de las Naciones Unidas.

Clemente, A., Arroyo, M.G., Zúñiga, R., Figueroa, A., Pérez, M.A., \& López, C.S. (2012). Relación de atenuación del movimiento del suelo para la aceleración máxima (PGA) sobre el cinturón volcánico mexicano (MVB); análisis por trayectoria: Guerrero-Querétaro. Ingeniería Sismica, 87 (1), 67-93.

Consejo Nacional de Evaluación de la Política de Desarrollo Social, CONEVAL (2016). Rezago social a nivel zonas urbanas (AGEB urbanas). Recuperado de http://www.coneval. gob.mx/Medicion/Paginas/Rezago_social_ AGEB_2010.aspx

Coy, M. (2010). Los estudios del riesgo y de la vulnerabilidad desde la geografía humana. Su relevancia para América Latina. Población y Sociedad, 17 (1), 9-28.

Cutter, S.L., Boruff, B.J, \& Shirley, W.L. (2003). Social Vulnerability to Environmental Hazards. Social Science Quarterly, 84 (2), 242-261. https://doi. org/10.1111/1540-6237.8402002

Eraso, M. (2008). Gestión del riesgo hídrico en comunidades vulnerables. Inundaciones en el arroyo El Cardalito, Mar del Plata, Buenos Aires. Revista Universitaria de Geografia, 17 (1), 285-307.

Feito, L. (2007). Vulnerabilidad. In Anales del Sistema Sanitario de Navarra, 30 (3), 7-22.

Filgueira, C. (2005). Estructura de oportunidades, activos de los hogares y movilización de activos en Montevideo (1991-1998). Prisma, 21 (1), 67-115.

Galassi, G. \& González, L.M. (2012). Vulnerability Factors in the Middle Class: Evidence for Argentina and Mexico after the crisis of the 1990s. Frontera Norte, 24 (47), 89-116.
García De León, A. (noviembre, 2006). Yucatán 1990-2000: evaluación del bienestar municipal y regional a partir de las condiciones físicas de las viviendas. En El nuevo debate sobre la región y el desarrollo regional: propuestas teóricas y metodológicas. S. Rodríguez (Coord. Gral.) Ponencia llevada a cabo en el $1^{\circ}$ Encuentro nacional sobre desarrollo regional en México. Recuperado de http://www.eumed. net/jirr/1/AMECIDER2006/PARTE\%20 $1 / 70 \% 20$ Armando $\% 20$ Garcia $\% 20$ de $\% 20$ Leon\%20Loza.pdf

García, N. \& Villerías, S. (2016). Niveles de vulnerabilidad social de la ciudad de Chilpancingo, Guerrero, México. Revista Geográfica de América Central, 1 (56), 259288.

García, N. \& Sánchez, Á. (2012). Changes in the sectoral orientations of the state of Guerrero between 1970 and 2000. Investigaciones Geográficas, 77 (1), 107123.

Golovanevsky, L. (2007). Vulnerabilidad y transmisión intergeneracional de la pobreza. Un abordaje cuantitativo para Argentina en el siglo XXI. (Tesis de Doctorado). Facultad de Ciencias Económicas, Universidad de Buenos Aires. Buenos Aires, Argentina.

González, L., Ortecho, M., \& Molinatti, F. (2013). Desde la vulnerabilidad a la movilidad social, con una mirada decolonial. Estudios. Centro de Estudios Avanzados. Universidad Nacional de Córdoba, 30 (1), 177-196.

Hufschmidt, G. (2011). A comparative analysis of several vulnerability concepts. Natural Hazards, 58 (2), 621-643. https:// doi.org/10.1007/s11069-011-9823-7

Instituto Nacional de Geografía y Estadística INEGI. (2016). Sistema estatal y municipal de base de datos. http://www.inegi.org.mx/

Juárez, C., Márquez, M., Salgado, S., Pelcastre, B.E., Ruelas, M.G., \& Reyes, H. (2014). La desigualdad en salud de 
grupos vulnerables de México: adultos mayores, indígenas y migrantes. Revista Panamericana de Salud Pública, 35 (4), 284-290.

Juárez, M.C., Iñiguez, L., \& Sánchez, M.A. (2006). Niveles de riesgo social frente a desastres naturales en la Riviera Mexicana. Investigaciones Geográficas, Boletín del Instituto de Geografía, 61 (1), 75-88.

Kaztman, R. (2000). Notas sobre la medición de la vulnerabilidad social. (Documentos de Trabajo). Comisión económica para América Latina y el Caribe. Ciudad de México, México.

Lampis, A. (2010). ¿Qué ha pasado con la vulnerabilidad social en Colombia? Conectar libertades instrumentales y fundamentales. Revista Sociedad y Economía, 19 (1), 229-261.

León, F.J. (2011). Pobreza, vulnerabilidad y calidad de vida en América Latina. Retos para la bioética. Acta bioeth, 17 (1), 19-29. https:// doi.org/10.4067/s1726569x2011000100003

Lin, K.H.E., Lee, H.C. \& Lin, T.H. (2017). How does resilience matter? An empirical verification of the relationships between resilience and vulnerability. Natural Hazards, 87 (2), 1-22. https://doi. org/10.1007/s11069-017-2916-1

Ministerio de Ambiente y Desarrollo Sustentable, MAyDS (2016). Sistema de Indicadores de Desarrollo Sostenible Argentina. Recuperado de http:// estadisticas.ambiente.gob.ar/archivos/web/ Indicadores/file/multisitio/publicaciones/ Indicadores_2015_internet.pdf

Morales, S., Rodríguez, M., \& Sánchez, E. (2013). Seguridad urbana y vulnerabilidad social en ciudad Juárez. Un modelo desde la perspectiva de análisis espacial. Frontera Norte, 25 (49), 29-56.

Paveglio, T. \& Edgeley, C. (2017). Community diversity and hazard events: understanding the evolution of local approaches to wildfire. Natural Hazards, 87 (2), 1.083-1.108. https:// doi.org/10.1007/s11069-017-2810-x

Pavarini, S.C.I., Barha, E.J., Mendiondo, M.S.Z.D., Filizola, C.L.A., Petrilli Filho, J.F., \& Santos, A.A.D. (2009). Family and social vulnerability: a study with octogenarians. Revista Latino-americana de Enfermagem, 17(3), 374-379. https://doi. org/10.1590/s0104-11692009000300015

Pizarro, R. (2001). La vulnerabilidad social y sus desafios: una mirada desde América Latina. Santiago de Chile: Naciones Unidas.

Propin, E. (2003). Teorías y métodos en Geografía Económica. México D.F.: Universidad Nacional Autónoma de México.

Propin, E. \& Sánchez, Á. (1998). Niveles de asimilación económica del estado de Guerrero. Investigaciones Geográficas, 37(1), 59-70.

Ramírez, M. T. (2011). Evidencias ambientales de cambios de nivel de la costa del Pacífico de México: terremotos y tsunamis. Revista de geografía de Norte Grande, 49, 99-124. https://doi. org/10.4067/s0718-34022011000200007

Ramírez, M. T. \& Lugo-Hubp, J. (2000). Efectos del sismo (7.0=Mw) del 15 de junio de 1999 en Puebla y estados vecinos. Investigaciones Geográficas, 43 (1), 32-41.

Rodríguez, A., Ruz, M., \& Hernández, B. (2012). Riesgo y vulnerabilidad en Llano Largo, Acapulco: la tormenta Henriette. Economía, Sociedad y Territorio, 12 (39), 425-447. https://doi.org/10.22136/ est00201277

Ruíz, M. (2012). Vulnerabilidad territorial frente a desastres naturales: el caso de la isla de Mallorca (Baleares, España). GeoFocus, 12(1), 16-52. 
Sales, F. J. (coord.) (2012). Pobreza y factores de vulnerabilidad social en México. México D.F.: Centro de estudios sociales y de opinión pública.

Sánchez D. \& Egea, C. (2011). Enfoque de vulnerabilidad social para investigar las desventajas socioambientales: su aplicación en el estudio de los adultos mayores. Papeles de Población, 17 (69), 152-185.

Sánchez, A. (2000). Marginación e ingreso en los municipios de México: análisis para la asignación de recursos fiscales. México D.F.: Miguel Ángel Porrúa, Instituto de investigaciones económicas, UNAM.

Secretaría de Medioambiente y Recursos Naturales, SEMARNAT (2000). La evaluación del impacto ambiental. Logros $y$ retos para el desarrollo sustentable 1995-2000. México: Instituto Nacional de Ecología.

Stern, C. (2004). Vulnerabilidad social y embarazo en adolescentes en México. Papeles de Población, 10 (39), 129-158.

Valenzuela, E. \& Coll-Hurtado, A. (2009). La construcción y evolución del espacio turístico de Acapulco (México). Anales de Geografia, 30 (1), 163-190.

Vázquez, V. (2007). Valoración de las condiciones naturales asociadas con el desarrollo de la actividad turística en el estado de San Luis Potosí, México. Espacio y Desarrollo, 19 (1), 93-103. 